events occur in vivo, it is not difficult to imagine how SIV $_{\text {SMM-PBil4 }}$ might rapidly establish an extensive and spreading infection. But the migration of virus-producing lymphocytes to the GALT, and their apparent sequestration there, cannot yet be readily explained. Because SIV $_{\text {SMM.PBi14 }}$ can induce cytokines such as interleukin-2 in cultured lymphocytes, it would not be far-fetched to propose that infected lymphocytes and monocyte-macrophage populations in lymphoid structures associated with the gastrointestinal tract secrete cytokines which influence the normal mononuclear-cell traffic in these tissues, or directly affect gastrointestinal physiology. One or more of the SIV $_{\text {SMM.PBil4 }}{ }^{-}$ encoded proteins, such as an envelope protein that has undergone mutation, could elicit the 'inappropriate' production and/or release of such regulatory factors.

Monkeys infected with $\operatorname{SIV}_{\text {SMм- } \mathrm{PB} \mid 14}$ apparently have high levels of circulating tumour necrosis factor (TNF), which may be another reflection of aberrant virusinduced cytokine release. Increased TNF production is also associated with HIV-1 infection of primary human monocytes? The rapid clinical deterioration of SIV $_{\text {SMM-PBi!4 }}$-infected macaques, which develop profuse bloody diarrhoea and an associated metabolic acidosis, is reminiscent of Gram-negative septicaemia, presumably also mediated by TNF (ref. 8).

Lentiviruses characteristically cause a mild primary syndrome, usually followed by a prolonged and unremitting secondary disease which may last for months to years. In man, acute HIV infection is associated with a 'mononucleosis-like' disease accompanied by cervical, occipital, axillary or even generalized lymphadenopathy during this early phase of infection in 75 per cent of cases 9 . Interestingly, diarrhoea has been reported in a third of recently infected patients ${ }^{10}$. The acute disease caused by feline immunodeficiency virus also results in pronounced generalized lymphadenopathy which centres on the gastrointestinal tract ${ }^{11}$. Thus the disease induced in monkeys by $\mathrm{SIV}_{\mathrm{SMM}-\mathrm{PB} \mathrm{i} 14}$ could represent an unusually aggressive and

1. Kestler, H. et al. Science 248, 1109-1112 (1990)

2. Dewhurst, S., Embretson, J.E., Anderson, D.C., Mullins, J.I. \& Fultz, P.N. Nature 345, 636-640 (1990).

3. Fultz, P.N. et al. Aids Res. hum. Retroviruses 5, 397-409 (1989).

4. McDonald, G.B. \& Sale, G.E. in The Pathology of Bone Marrow Transplantation (eds Sale, G.E. \& Shulman H.M.) 77-103 (Year Book Medical, Chicago, 1984)

5. Ferguson, A. in Immunological Aspects of the Liver and the Gastrointestinal Tract (eds Ferguson, A. \& MacSween, R.N.M.) 152 (MTP, Lancaster, 1967).

6. MacDonald, T.T. \& Spencer, J. J. exp. Med. 167, 1341 1349 (1988).

. Merrill, J.E., Koyanagi, Y. \& Chen, I.S.Y. J. Virol. 63 4404-4408 (1989)

8. Tracy, K.J. et al. Science 234, 470-474 (1986).

9. Tindall, B., Cooper, D.A., Donovan, B. \& Penny, R. in Infectious Disease Clinics of North America - Medical Management of AlDS (eds Sande, M.A. \& Volberding, P.A.) 329-351 (W.B. Saunders, Philadelphia, 1988)

10. Cooper, D. A. et al. Lancet I, 537-540 (1985).

11. Yamamoto, J. K. et al. Am. J. vet. Res. 49, 1246-1258 (1988) highly aberrant primary lentivirus infection which efficiently and rapidly targets infected cells to lymphoid tissue in the digestive tract and results in death due to the loss of fluid and electrolytes.

The two new reports of infectious, disease-causing molecular clones of SIV also raise an interesting side issue pertaining to vaccine development. Fultz and her colleagues reported last year that asymptomatic sooty mangabey monkeys, previously infected with SIV $_{\text {SMM }}$, are completely protected from $\mathrm{SIV}_{\mathrm{SMM} \cdot \mathrm{PB}_{14}}$ induced disease ${ }^{3}$. The two sero-positive monkeys challenged with SIV $_{\text {SMM.PBjI4 }}$ did not die or show any signs of secondary disease. Although Desrosiers and his group have not carried out a similar experiment, they have isolated several molecular clones of SIV $\mathrm{VAC}_{\mathrm{MAC}}$, in addition to the disease-producing SIV $_{\text {MAC-239 }}$ clone, which give rise to persistent virus infections; so far, however, the avirulent clones have failed to induce immunodeficiency in inoculated macaques. One wonders whether avirulent variants of HIV that can replicate efficiently in vivo, and elicit similar protective immunity in man, might also be isolated or even constructed in the laboratory.

Malcolm A. Martin is in the Laboratory of Molecular Microbiology, National Institute of Allergy and Infectious Diseases, Bethesda, Maryland 20892, USA

\title{
Blocks on the viral exit
}

\section{Jonathan Weber}

Writing in News and Views nearly two years ago, David Baltimore ${ }^{1}$ coined the term 'intracellular immunization' to describe the prospect of using a dominant negative mutant viral gene to interfere with the replication of wild-type virus. The most attractive early application of this approach to cell protection is against HIV infection - both because of the pandemic nature of this lethal virus, and the possibility of relatively easy access to its target, the $\mathrm{CD}^{+}$peripheral blood mononuclear cells. Three candidate HIV genes in which mutations can be made to give a dominant negative phenotype have already been described: gag mutants, which probably interfere with HIV replication at the level of virus assembly ${ }^{2}$, and tat and rev transdominant mutants ${ }^{3,4}$. Now, on page 625 of this issue Buonocore and Rose ${ }^{5}$ describe a novel approach to 'intracellular immunization' using a CD4 mutant that is retained by the endoplasmic reticulum and that prevents transport of the HIV envelope proteins to the cell surface.

\section{Role of CD4}

That the CD4 molecule is the cellular receptor for HIV has been known since 1984, and soluble recombinant CD4 (srCD4) neutralizes HIV infection of target cells in vitro, and probably in vivo. But less attention has been paid to the role of CD4 in the transport of HIV env proteins and the production of mature viral particles. Asjo and colleagues ${ }^{6}$ showed that $\mathrm{CD} 4$ receptor density on diverse monocytoid cell line clones from the U937 parental line was associated with the quantity of infectious virus produced; Hoxie et al. $^{7}$, and subsequently Stevenson et al. ${ }^{8}$, demonstrated that down-regulation of CD4 post-HIV infection was associated with intracellular complexing of $\mathrm{CD} 4$ with HIV envelope protein. However, HIV env proteins can clearly be expressed in CD $4^{-}$cells, as shown by the Chinese hamster ovary cell line production of the HIV envelope glycoprotein gp120. The role of CD4 in the normal transport of gp120 requires further study.

Buonocore and Rose generated a mutant form of soluble CD4 (sCD4), which contains the sequence Lys-AspGlu-Leu (KDEL) at the C terminus; this sequence is a signal that can retain soluble proteins within the lumen of the endoplasmic reticulum". Constructs of sCD4KDEL were expressed in HeLa cells, with and without co-expression of gp120 and gp160. The sCD4-KDEL mutant prevented expression of gp120 on the cell surface, whereas normal SCD4 alone did not. In addition, the sCD4-KDEL mutant did not interfere with the normal surface expression of CD4 in the same cell. Using an assay for syncytium formation in HeLa CD4 $4^{+}$cells, Buonocore and Rose showed that sCD4-KDEL blocks syncytia formation after gp120/gp41 transfection. Theoretically, cells transfected with sCD4-KDEL should be protected from the consequences of HIV infection, while CD4 production and expression remains normal.

This approach to intracellular protection is elegant, and it avoids the use of dominant negative mutants based on viral genes which could lead to evasion of the block by further mutation. Any mutation of HIV env that prevents binding to SCD4-KDEL should reduce the binding of HIV to its receptor, and successful escape mutants from sCD4-KDEL should also be unable to infect $\mathrm{CD} 4^{+}$cells. But this study has not shown that wildtype HIV replication can be suppressed by sCD4-KDEL, nor that $T$ cells transfected with $s C D 4-K D E L$ are functionally normal. It remains possible that sCD4-KDEL could become saturated by 
gp120 within the endoplasmic reticulum, thereby by-passing the block; or that sCD4-KDEL escape mutants with lower affinity for $\mathrm{CD} 4$, but still retaining their tropism for CD4 cells, could be formed. These practical issues can be addressed only in an active HIV replication system.

\section{Second generation therapy}

Every week I watch AIDS patients deteriorate and waste away despite Zidovudine (ZDV) therapy. They urgently need novel therapies that act at different sites. Buonocore and Rose's report adds to the long list of potential AIDS therapies, and targets based on delivery of novel or mutated genes extend even beyond the mutant viral genes, into 'suicide' genes, or delivery of antisense RNA. If the reverse transcriptase inhibitors - both ZDV and the up-coming dideoxyinosine (DDI) - belong to the first generation of therapy, then the second generation will be drugs active at new sites. The entry of HIV is blocked by soluble recombinant CD4 in vivo, but at the cost of high doses of immunogenic recombinant protein; between 15 and 20 per cent of patients already have antisCD4 antibodies, and more will develop them after therapy ${ }^{10}$. The polysulphated polysaccharides - active in vitro, but seen as failures in a clinical trial because they did not act when administered orally are now attracting renewed interest. They block entry of all HIV isolates and their principal toxicity (anti-coagulant activity) may be chemically separated from their anti-viral activity; at least one of these drugs may go back into clinical trials, this time through a more appropriate route of administration, as they are not absorbed when taken orally. Meanwhile, pharmacokinetic data on the HIV protease inhibitor peptides are eagerly awaited pending clinical studies.

It is unlikely that second-generation anti-retroviral therapy will be based on 'intracellular immunization'. But if the techniques can be refined, then transfection of an SCD4-KDEL construct into bone-marrow stem cells might be a very attractive third-generation option.

Jonathan Weber is in the Infectious Diseases Unit, Royal Postgraduate Medical School, Hammersmith Hospital, Du Cane Road, London W12 ONN, UK.

1. Baltimore, D. Nature 335, 395-396 (1988)

2. Trono, D., Feinberg, M. \& Baltimore, D. Cell 59, 113120 (1989).

3. Green, M., Ishino, M. \& Loewenstein, P. Cell 58, 215 233 (1989).

4. Malim, M., Bohlein, S., Hanber, J. \& Cullen, B. Cell 58 205-214 (1989).

5. Buonocore, L. \& Rose, J. K. Nature 345, 625-628 (1990).

6. Asjo, B. et al. Virology 157, 359-365 (1987).

7. Hoxie, J. et al. Science 229, 1123-1127 (1986)

8. Stevenson, M. et al. Cell 53, 483-496 (1988).

9. Munro, S. \& Pelham, H.R.B. Cell 48, 899-907 (1987).

10. Schooley, R. et al. Ann. intern Med. 112, 247-253 (1990).

\section{Running around in circles}

\section{Yoseph Imry}

IT is well-known that currents that 'never decay' can flow in superconductors, but less familiar is the prediction' that such persistent currents can also be induced by magnetic fields in normal (non-superconducting) systems, with the proviso that they be small enough for quantum mechanics to apply. Levy et al. ${ }^{2}$ now provide evidence for the existence of such currents, and moreover on the mesoscopic rather than microscopic scale.

Levy et al. have used electron-beam techniques to fabricate a system of $10^{7}$ square-shaped copper rings, 0.55 micrometres on a side. They employed a SQUID (superconducting quantum interference device) to measure the magnetic moment (proportional to the circulating currents) induced in the system at very low temperatures by a magnetic field applied

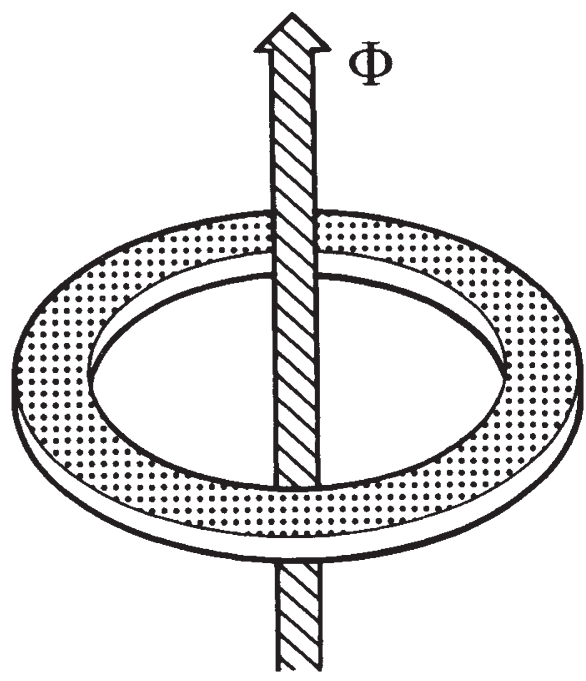

A conducting ring threaded by a magnetic flux $\Phi$.

perpendicular to the plane of the rings. The magnetic signal was picked out from the background by harmonic generation techniques which rely on the specific nonlinear periodic response of the rings. The induced currents did not decay, at least on the timescale of the inverse frequency used in the experiment (a fraction of a second). Such times are huge on a microscopic scale, and are sufficient to qualify the currents as 'persistent'. The magnitude of the observed signal is consistent with theory within an order of magnitude, on the assumption that the signals from all rings add coherently ${ }^{3}$.

Despite theoretical expectations ${ }^{1.4 h}$, there has been some debate about whether persistent currents can be maintained in a system of this sort. In classical systems, circulating currents must decay. But quantum mechanics permits a magnetic field to induce persistent dia- magnetic moments associated with orbital electrons (in atoms and molecules, for example). In the experiments of Levy et al. these moments are associated with persistent currents that circulate around the rings. In macroscopic systems they may be expected to persist only when quantum effects still apply (in superconductivity, for example); so the existence of the effect in 'normal' mesoscopic systems was not generally accepted.

The circulating currents depend in a periodic way on the magnetic flux through the ring, being a manifestation of the quantum-mechanical Aharonov-Bohm effect? This is a counter-intuitive phenomenon in which charged particles encircling a line of magnetic flux are influenced by the magnetic field even though they never pass directly through the flux line. The effect requires that the phase of the particles' wavefunction stays well defined for at least one complete revolution of a particle around the ring.

The persistent equilibrium current $I$ in a ring threaded by a flux $\Phi$ (see figure) is proportional to $\partial J / \partial \Phi$, where $J$ is the thermodynamic potential (for example, the ground-state energy for a closed system at zero temperature). Thus $I$ is non-zero if $J$ depends on $\Phi$. The flux $\Phi$ induces a phase change $\phi=2 \pi \Phi / \Phi_{0}$ in the wavefunction of an electron that circulates once around the ring, so $I$ is a periodic function ${ }^{8.9}$ of $\Phi$ with period $\Phi_{0}$. (Here $\Phi_{0}$ is the quantum flux unit h/e, where $h$ is Planck's constant and $e$ is the charge of an electron.) For the wavefunction to stay coherent around the ring, the electron must be able to circulate around it without being scattered inelastically (that is, without other degrees of freedom becoming excited). Also, the thermal energy must be negligible and the charge carriers must not become localized in some small part of the ring. Weak, purely elastic scattering of the electrons (by defects, for example) is not detrimental to the persistent current.

Although quantum mechanics ensures the survival of the persistent currents, their magnitude depends on the microscopic arrangement of defects in each specific sample. The fundamental harmonic of the oscillatory persistent current corresponds to the AharonovBohm oscillation, characterized by the quantized period $\Phi_{0}$. That the conductance of such rings also exhibits such an oscillation is now widely known. The fundamental vanishes when the current is averaged over the different arrangements of defects in an 'ensemble' of systems that are identical on a macroscopic scale (Y. 